\title{
Changes in Mechanical Characteristics of Pre-Annealed Wires of Cu-Sn Alloy Manufactured by Continuous Draw Bending
}

\author{
Junichiro Tokutomi ${ }^{1}$, Kenichi Hanazaki ${ }^{1}$, Nobuhiro Tsuji ${ }^{2}$ and Jun Yanagimoto ${ }^{3}$ \\ ${ }^{1}$ Yazaki Technology Center, Yazaki Corporation, Susono 410-1194, Japan \\ ${ }^{2}$ Department of Materials Science and Engineering, Graduate School of Engineering, Kyoto University, Kyoto 606-8501, Japan \\ ${ }^{3}$ Institute of Industrial Science, The University of Tokyo, Tokyo 153-8505, Japan
}

\begin{abstract}
The changes in mechanical properties and microstructures of fine $\mathrm{Cu}-\mathrm{Sn}$ alloy wires manufactured by deep wire drawing and heat treatment, and of the wires deformed by draw bending were systematically investigated. During draw bending, the wires were subjected to the compressive/tensile or tensile/compressive strain in the longitudinal direction. As a result, it was confirmed that the softening caused by plastic deformation was induced when easing up the wire drawing strain by heat treatment were few, such as the decreasing strength and increasing elongation. Work hardening was induced when easing up the wire drawing strain by heat treatment were large, such as the increasing strength and decreasing elongation. With decreasing strength of softening induced by plastic deformation, increase in grain size is smaller than the strength deterioration. Additionally, it was confirmed that the occupancy probability of high-angle grain boundaries increases. This can be understood by considering that the many dislocations formed inside the grains are piled up in one direction during draw bending. Additionally, it is considered that ductility enhancement is caused by the increase in work hardening rate induced by the piled up dislocations.

[doi: $10.2320 /$ matertrans.MD201114]
\end{abstract}

(Received August 1, 2011; Accepted September 20, 2011; Published November 2, 2011)

Keywords: bending, mechanical properties, annealing, microstructure, fine copper wire

\section{Introduction}

For applications in automobiles and the flexural area of home electrics, fine wires are more and more desired. However, there is a risk of breakage during the manufacture and usage of the fine wires, since the cross-sectional area of the wires is extremely small. Consequently, high strength and large ductility are desirable for fine wires of $\mathrm{Cu}$ and $\mathrm{Cu}$ alloys.

There are several strengthening methods for metallic materials, such as dislocation strengthening, solid-solution strengthening, precipitation strengthening, dispersion strengthening, and grain refinement strengthening. The grain refinement method has attracted a considerable attention because of the improvement of recyclability and the decrease in environmental impact caused by less alloying elements.

Recently, many investigations have been performed on severe plastic deformation (SPD) processes, such as high pressure torsion, ${ }^{1,2)}$ equal channel-angular pressing, ${ }^{3,4)}$ and, accumulative roll bonding, ${ }^{5-9)}$ for manufacturing ultrafine grained metallic materials with grain sizes smaller than $1 \mu \mathrm{m}$, as reviewed by Azushima et al. ${ }^{10)}$ It has been also suggested that the conventional cold wire drawing process has the same nature as the SPD processes when the total imposed strain used to manufacture fine wires are greater than 4.0. ${ }^{11)}$ The mechanism of strength improvement in the ultrafine grained materials has been clarified, but that of ductility improvement is still unclear.

In the previous study, we have proposed the "continuous bending-drawing (CBD)" method involving a combination of continuous wire drawing (WD) with a large area reduction and subsequent draw bending in the final step, that produces fine wires. ${ }^{12)}$ It was confirmed that softening occurred when the wires are subjected to draw bending after WD. In particular, it was noteworthy that the total elongation of draw bended wire was $48 \%$ larger than that before the draw bending.
In this study, we investigate the changes in the mechanical properties of wires processed by WD and annealed and then draw bending, and also discuss the origins of mechanical properties.

\section{Experimental}

\subsection{CBD process and materials}

A dilute $\mathrm{Cu}-\mathrm{Sn}$ alloy was used in this investigation. The chemical composition of the alloy is shown in Table 1. As shown in this table, this alloy was contained only a small amount of $\mathrm{Sn}$ as the alloying element. This alloy was the same as that used in our previous study. ${ }^{12)}$ Figure 1 shows a schematic illustration of the sample setup used in this investigation. The initial rod of this alloy was prepared by casting, grooved rolling and annealing, and then the internal strain was completely removed by first annealing. The rods were elongated by WD in multi passes. It was then subjected to WD at room temperature with lubrication. The diameter of the initial rod was $7.7 \mathrm{~mm}$. The diameter of the fine wire after 23 passes of WD was $210 \mu \mathrm{m}$; thus, the maximum total area reduction was $99.93 \%$, which corresponded to the total equivalent strain of 7.2. Here, SPD by WD is imposed on the copper wire to form an ultrafine grained lamellar microstructure, is strengthening. The wire specimen was strainrelieved by final annealing to obtain the desired mechanical characteristics. The final annealing conditions and definitions of the various wire specimen name are shown in Table 2. Draw bending was performed to induce plastic deformation

Table 1 Chemical composition of the $\mathrm{Cu}-0.3$ mass $\% \mathrm{Sn}$ alloy studied (mass\%).

\begin{tabular}{cccccccc}
\hline & $\mathrm{O}$ & $\mathrm{As}$ & $\mathrm{Sn}$ & $\mathrm{Fe}$ & $\mathrm{P}$ & $\mathrm{Pb}$ & $\mathrm{Cu}$ \\
\hline $\mathrm{Cu}-0.3$ mass$\% \mathrm{Sn}$ & 0.014 & $<0.001$ & 0.266 & $<0.001$ & 0.001 & $<0.001$ & Bal. \\
\hline
\end{tabular}




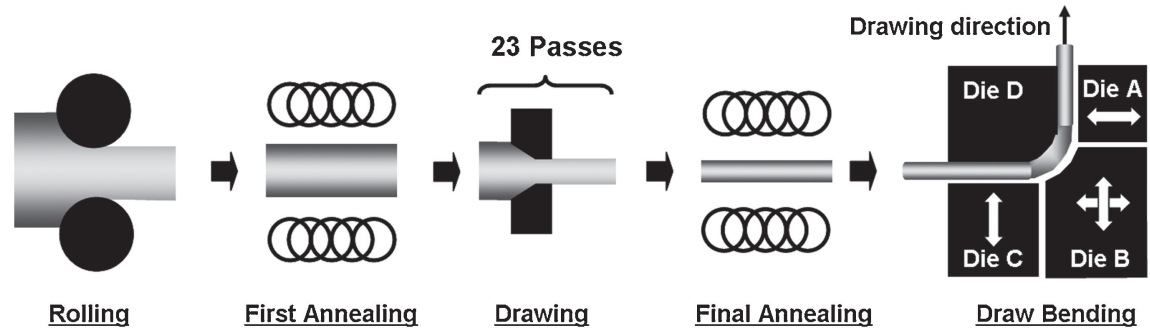

Fig. 1 Schematic diagram showing sample setup during CBD.

Table 2 Sample name and the final annealing conditions.

\begin{tabular}{lcc}
\hline \multicolumn{1}{c}{ Sample name } & \multicolumn{2}{c}{ Final annealing condition } \\
\cline { 2 - 3 } & Temperature & Time \\
\hline $\begin{array}{l}\text { Fully annealed specimen } \\
\left(\varepsilon^{\mathrm{WD}}=0.0\right)\end{array}$ & $673 \mathrm{~K}$ & $600 \mathrm{~s}$ \\
$1 / 2 \mathrm{EL}$ specimen & $623 \mathrm{~K}$ & $600 \mathrm{~s}$ \\
$1 / 4 \mathrm{EL}$ specimen & $623 \mathrm{~K}$ & $360 \mathrm{~s}$ \\
$1 / 2 \mathrm{TS}$ specimen & $623 \mathrm{~K}$ & $60 \mathrm{~s}$ \\
$3 / 4 \mathrm{TS}$ specimen & $573 \mathrm{~K}$ & $240 \mathrm{~s}$ \\
WD specimen & & - \\
$\left(\varepsilon^{\mathrm{WD}}=7.2\right)$ & - & \\
\hline
\end{tabular}

in different loading directions to control the strength and elongation of the fine wire, which was installed in the final step of the CBD process. Draw bending was not continuous to WD and the final annealing process. In Fig. 1, the arrangement of the draw bending die, $\mathrm{D}$ is a fixed die, whereas the positions of dies $\mathrm{A}, \mathrm{B}$ and $\mathrm{C}$ can be changed to control the bending radius. The wire was drawn in the Y-direction at a relatively low speed of $100 \mathrm{~mm} / \mathrm{min}$. The bending strain $\left(\varepsilon^{\text {bending }}\right)$ was estimated using an equation, ${ }^{12)}$ which is shown as

$$
\varepsilon^{\text {bending }}=\ln \left(1+\frac{d_{\mathrm{a}}}{R_{\mathrm{n}}}\right)\left(1+\frac{d_{\mathrm{b}}}{R_{\mathrm{n}}}\right) .
$$

Here, $R_{\mathrm{n}}$ is the curvature radius at the neutral plane of the wire specimen, and $d_{\mathrm{a}}$ and $d_{\mathrm{b}}$ are the radii of the wire specimen before and after bending, respectively. Draw bending was executed with a curvature radius of $0.85 \mathrm{~mm}$; thus, corresponding to an equivalent bending strain of 0.23 , which was selected to realize the best strength-ductility balance from the experimental result in our previous study. ${ }^{12)}$

\subsection{Evaluation method}

A tensile test of the wire product after the final annealing and draw bending processes was carried out at a nominal strain rate of $3 \times 10^{-3} \mathrm{~s}^{-1}$ at room temperature. The strength of the wire specimens were measured using an Instron-type testing machine with cord and yarn grips, which prevented jaw breaks of the wire specimens. The elongation of wires was measured using a video extensometer using two foam polystyrene balls with slits as markers. The initial gauge length was $50 \mathrm{~mm}$.

The microstructural and crystallographic features of the wire specimens after the final annealing and draw bending processes were characterized by electron backscatter diffraction (EBSD) measurement using a field-emission-type
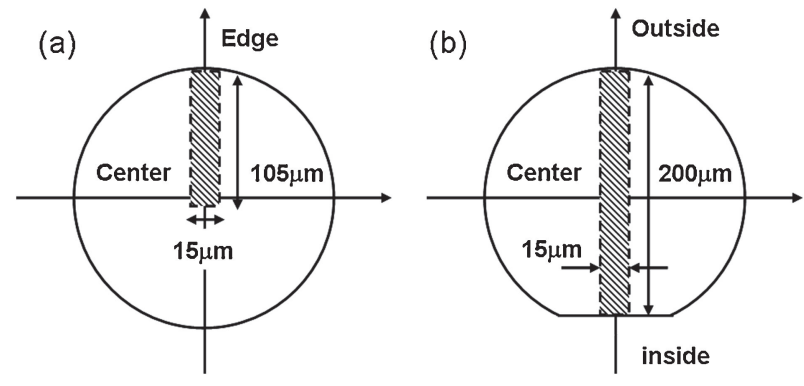

Fig. 2 Schematic diagram showing the EBSD measurement area on the cross section of a wire specimen after the final annealing and draw bending.

scanning electron microscope operated at $25 \mathrm{kV}$ equipped with a TSL orientation imaging microscopy system. Figure 2 shows the measurement area on the cross section of a wire specimen. The microstructures were measured from the center to the edge on the cross section of the specimen after final annealing using mapping areas of $15 \times 105 \mu \mathrm{m}^{2}$. The microstructures of the draw bending specimens were measured from the inside to the outside bending on the cross section of the specimen using mapping areas of $15 \times 200 \mu \mathrm{m}^{2}$. The cross section of the wire specimen was polished by an ion-beam polishing (JEOL) at $4.5 \mathrm{kV}$ for $8 \mathrm{~h}$ in argon gas atmosphere.

\section{Results}

\subsection{Changes in mechanical properties during final annealing and draw bending}

Figure 3 shows the nominal stress-strain curves of the wire specimens after various final annealing conditions. The ultimate tensile stress (UTS) of the fully annealed $\left(\varepsilon^{\mathrm{WD}}=0.0\right)$ and WD $\left(\varepsilon^{\mathrm{WD}}=7.2\right)$ specimens reaches approximately $220 \mathrm{MPa}$ and $790 \mathrm{MPa}$, respectively. The strength of the WD specimen is about four times higher than that of the fully annealed specimen. The $1 / 2 \mathrm{TS}$ specimen exhibits UTS of approximately $525 \mathrm{MPa}$, which is almost $66 \%$ lower than that of the WD specimen. The total elongation of the fully annealed specimen is $22.8 \%$. The total elongation of the WD specimen is $2.6 \%$, and then that of the $1 / 2 \mathrm{TS}$ specimen substantially decreases to $1.05 \%$. Thereafter, it was confirmed that ductility greatly increased with the saturation of decreasing stress.

Figure 4 shows the nominal stress-strain curves of the wire specimens after draw bending $\left(\varepsilon^{\text {Bending }}=0.23\right)$. The UTS of the fully annealed and WD specimens processed by draw bending reaches approximately $270 \mathrm{MPa}$ and $690 \mathrm{MPa}$, 


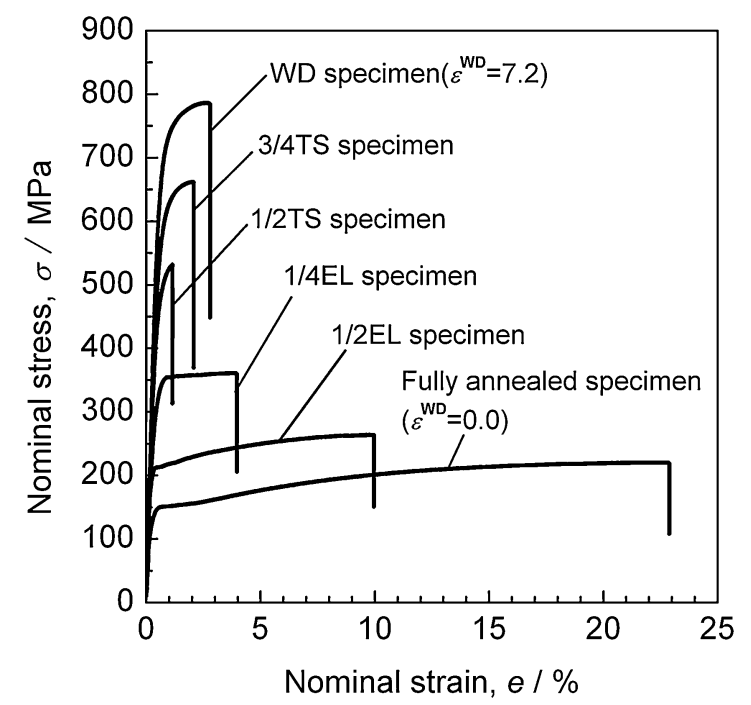

Fig. 3 Nominal stress-strain curves of $\mathrm{Cu}-0.3$ mass $\% \mathrm{Sn}$ alloy wire specimens after final annealing.

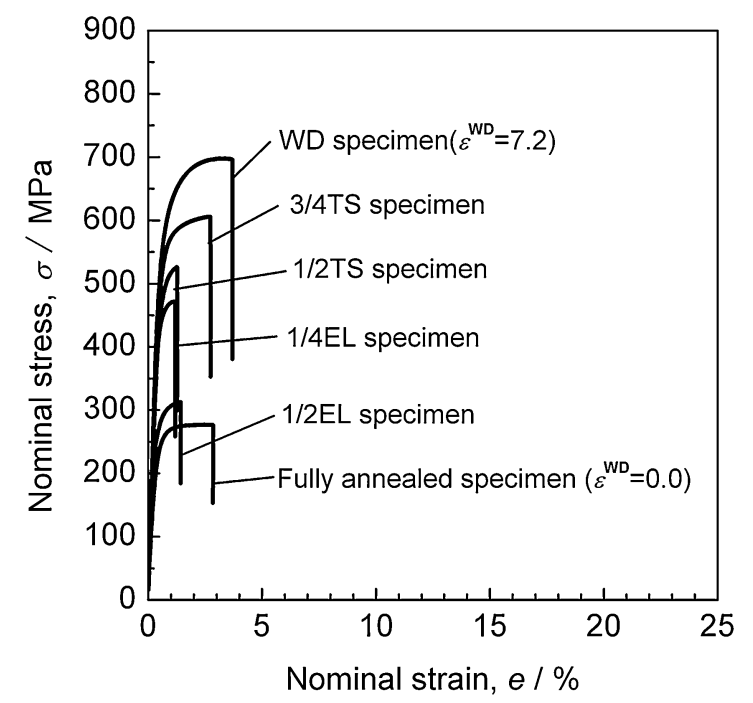

Fig. 4 Nominal stress-strain curves of the $\mathrm{Cu}-0.3$ mass\%Sn alloy specimens draw bending processed to an equivalent bending strain of 0.23 after final annealing.

respectively. The $1 / 2 \mathrm{TS}$ specimen processed by draw bending exhibits UTS of approximately $520 \mathrm{MPa}$. The total elongation of the fully annealed specimen after draw bending is $2.85 \%$. The total elongation of the WD specimen after draw bending is $3.69 \%$, and then that of the $1 / 2 \mathrm{TS}$ specimen decreases to $1.26 \%$.

The UTS and total elongation of the wire specimens obtained from the stress-strain curves showing in Figs. 3 and 4 are summarized in Fig. 5, which also expresses a strength-ductility balance of the specimens. Deformationinduced softening ${ }^{12}$ ) is observed in the wire specimens deformed by draw bending, particularly in the WD and 3/4TS specimens where decrease in the strength and increase in the ductility are significant. Work hardening is observed from $1 / 4 \mathrm{EL}$ to fully annealed specimens deformed by draw bending. It was confirmed that deformation-induced softening and work hardening flips around 1/2TS specimen.

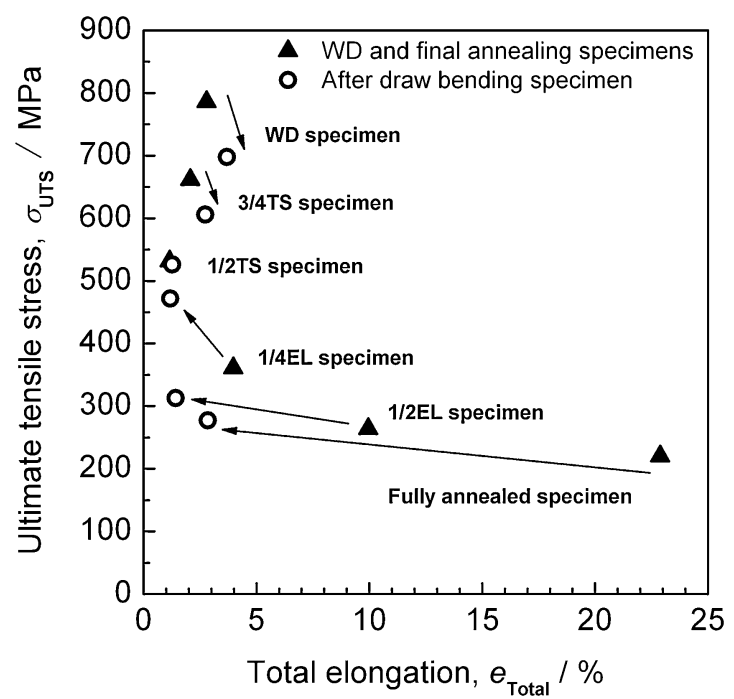

Fig. 5 Changes in mechanical behaviors of wire specimens processed by draw bending.

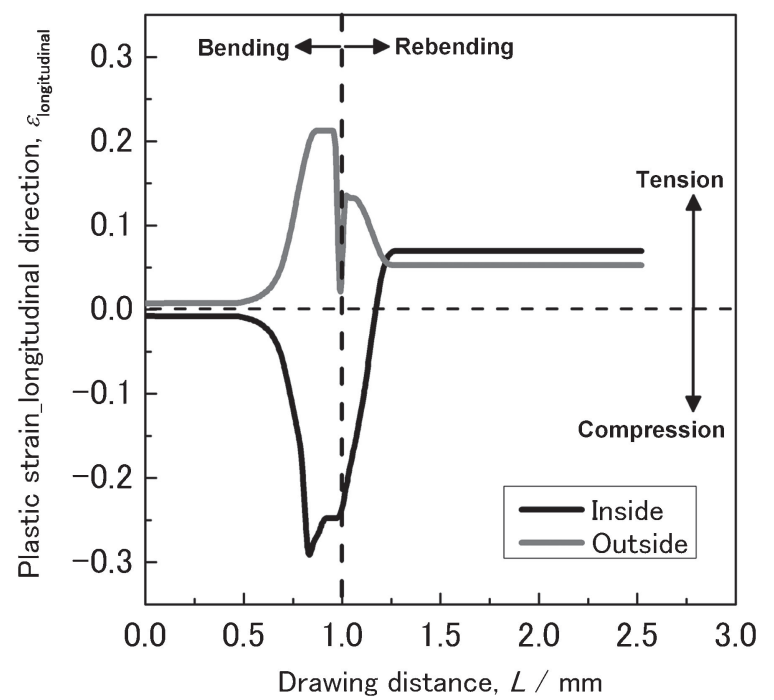

Fig. 6 Results of FEM simulation showing applied plastic strain in longitudinal direction.

\subsection{FEM analysis of draw bending}

The plastic strain in a wire specimen during draw bending was estimated by a finite element method (FEM). The wire specimen was constructed with 3D solid elements, and the simulation was carried out using ANSYS Mechanical. The wire model used had a continuous strain distribution, and precise material properties were inputted. Drawing displacement was applied in the longitudinal direction of wire specimen, and the radius direction was not constrained. The mass density, Young's modulus, Poisson's ratio and friction coefficient between the die and the wire specimen used in the FEM simulation were $8.9 \times 10^{3} \mathrm{~kg} / \mathrm{m}^{3}, 130 \mathrm{GPa}, 0.33$ and 0.05 , respectively. The true stress-strain curve of the WD specimen obtained from the tensile test shown in Fig. 3 was used as the material property.

Figure 6 shows the FEM simulation result of the plastic strain in the longitudinal direction of the WD specimen 

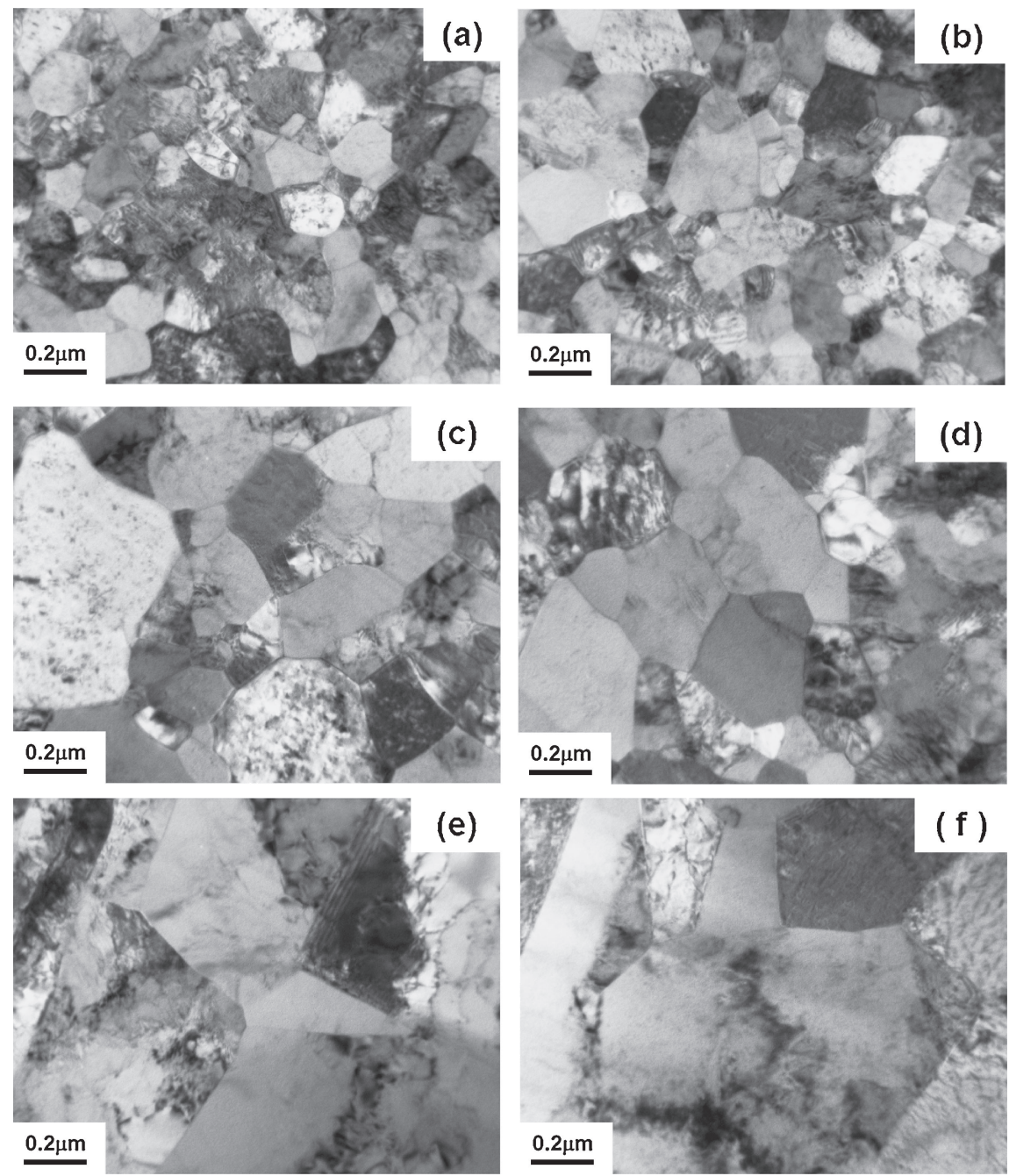

Fig. 7 TEM micrographs of the wire specimens annealed under various final annealing conditions and processed by draw bending: (a) WD specimen, (b) WD specimen after draw bending, (c) 1/2TS specimen, (d) 1/2TS specimen after draw bending, (e) fully annealed specimen, and (f) fully annealed specimen after draw bending.

during draw bending. As for the inside bending (called Inside), a compressive plastic strain of approximately $\varepsilon_{\mathrm{x}}=0.27$ is applied in the bending range, and then the tensile plastic strain of approximately $\varepsilon_{\mathrm{x}}=0.33$ is applied within the rebending range. Simultaneously, as for the outside bending (called Outside), a tensile plastic strain of approximately $\varepsilon_{\mathrm{x}}=0.21$ is applied in the bending range, and then the compressive plastic strain of approximately $\varepsilon_{\mathrm{x}}=0.16$ is applied within the rebending range. It was confirmed that continuous strain reversal was induced by draw bending to the wire specimen.

\subsection{Changes in texture before and after draw bending}

Figure 7 shows TEM micrographs of the wire specimens annealed under various final annealing conditions and then processed by draw bending. The microstructures are observed without being tilted from the cross-section of the wire specimens. The WD specimen [Fig. 7(a)] has ultrafine grains with dislocation substructures, and the processed draw bending specimen [Fig. 7(b)] shows a slight increase in grain size. In the $1 / 2 \mathrm{TS}$ specimen [Fig. 7(c)], the grain size increases and the recovery seems to occur inside the grains, compared with those observe in the WD specimen. In the $1 / 2$ TS specimen processed by draw bending [Fig. 7(d)], the change in microstructure is not confirmed. The occurrence of recrystallization is confirmed and few dislocation substructures are observed inside the grain in the fully annealed specimen [Fig. 7(e)]. Moreover, the fully annealed specimen processed by draw bending [Fig. 7(f)] has grains with many dislocation substructures.

The grain boundaries and dislocations inside the grains are classified into high-angle grain boundaries (HAGBs) and low-angle grain boundaries (LAGBs); the HAGBs have misorientations larger than 15 degrees, and the LAGBs have misorientations ranging from 2 to 15 degrees. The boundaries having misorientations smaller than 2 degrees are cut off so as to remove inaccuracies in EBSD measurement and analysis. Figure 8 shows the fraction of HAGBs obtained by EBSD analysis for the wire specimens processed under various final annealing and draw bending conditions. As for the WD and $1 / 2 \mathrm{TS}$ specimens after draw bending, the fraction of HAGBs increases than after the final annealing. As for the 1/4EL and fully annealed specimens after draw bending, the fraction of HAGBs decreases than after the final annealing. 


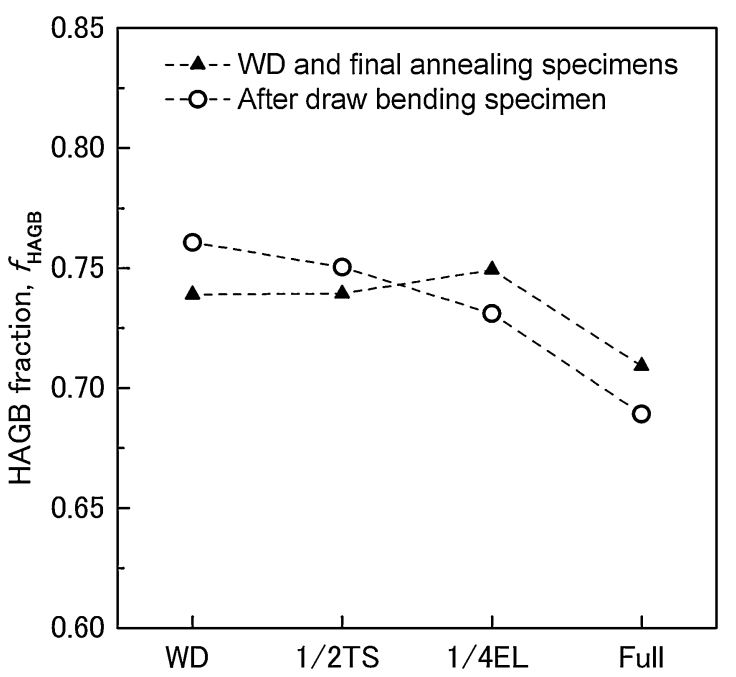

Fig. 8 Changes in HAGB fractions of the wire specimen after final annealing and draw bending: (a) from inside to center and (b) from outside to center.

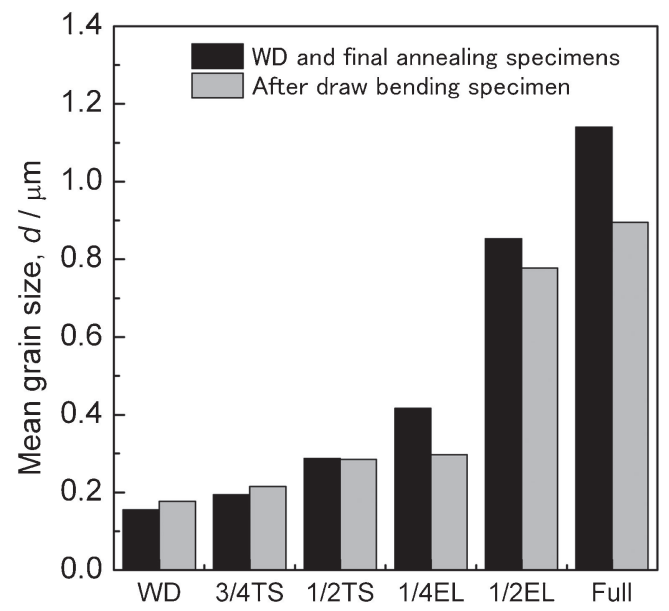

Fig. 9 Changes in grain size after final annealing and draw bending.

\section{Discussion}

\subsection{Microstructure and tensile strength}

In this section, the relationship between the strength and structure of copper is discussed. The Hall-Petch equation is commonly used for quantitative evaluation of the relationship between the strength and grain size of metallic materials, ${ }^{13-15}$ ) which is shown as

$$
\sigma_{\mathrm{y}}=\sigma_{0}+k d^{-1 / 2} .
$$

Here, $\sigma_{0}$ is the friction stress, $k$ is the Hall-Petch coefficient and $d$ is the grain size and $\sigma_{\mathrm{y}}$ is the yield stress. In eq. (2), the strength is shown to be proportional to the minus square root of the grain size. The experimental data about the grain size obtained in this study are shown in Fig. 9. It was confirmed that the grain size changed from 0.15 to $1.14 \mu \mathrm{m}$ after final annealing and from 0.17 to $0.89 \mu \mathrm{m}$ after draw bending. Specifically, the change in grain size was smaller than the deterioration of strength in the WD and 3/4TS specimens; the decrease in grain size was just $0.02 \mu \mathrm{m}$, while the deterioration of strength was approximately $100 \mathrm{MPa}$. It should be noted that the WD and 3/4TS specimens have dislocation substructures inside the ultrafine grains. The mismatch between the grain size and strength might be affected by the dislocation strengthening mechanism. ${ }^{16)}$ The Bailey-Hirsch equation is commonly used for quantitative evaluation of the relationship between strength and dislocation density, which is shown as

$$
\Delta \tau=\alpha \mu b \sqrt{\rho}
$$

Here, $\alpha$ is the constant, $\mu$ is the shear modulus, $b$ is the magnitude of the Burgers vector and $\rho$ is the dislocation density. The change in the nominal stress produced by dislocation to eq. (4).

$$
\Delta \sigma=M \Delta \tau
$$

Here, $M$ is the Taylor factor. The $M$ value was measured by using OIM Analysis after the EBSD pattern had been acquired by using OIM Data Collection. The $M$ values obtained after the final annealing and draw bending of the WD specimen were 3.14 and 3.15, and those obtained after the final annealing and draw bending of the $3 / 4 \mathrm{TS}$ specimen were 3.07 and 3.08, respectively. After draw bending, no substantial change in $M$ was observed. Consequently, the main cause of the deterioration of strength in the WD and $3 / 4 \mathrm{TS}$ specimens can be considered as decrease in dislocation density from eqs. (3) and (4).

\subsection{Plastic instability of wire specimens}

The mechanical behaviors of the specimens before and after draw bending, specially ductility improvement could be explained simply by plastic instability conditions. Plastic instability conditions, ${ }^{17,18)}$ in other words, the conditions of necking propagation in the tensile test, are expressed by

$$
\frac{\mathrm{d} \sigma}{\mathrm{d} \varepsilon} \leq \sigma
$$

Here, $\sigma$ is the flow stress, $\varepsilon$ is the true strain, and $\mathrm{d} \sigma / \mathrm{d} \varepsilon$ is the work hardening rate.

Figure 10 shows the true stress-strain curves and work hardening rate of the wire specimens annealed at various temperatures and the wire specimen processed by draw bending. The dashed and solid lines show the true stressstrain curve and work hardening rate of each wire specimen, respectively. For the WD specimen processed by draw bending, the flow stress and work hardening rate were respectively lower and higher than those for the WD specimen. On the other hand, for the fully annealed specimen processed by draw bending, the flow stress and work hardening rate were respectively higher and lower than those for the fully annealed specimen. The work hardening rate rapidly decreased at a very early stage of tensile deformation. As shown in eq. (5), when the work hardening rate is higher than flow stress, it means uniform elongation continues. Consequently, it is considered that the ductility improvement in the WD specimen after draw bending occurs due to increase in the uniform elongation than for the WD specimen.

\subsection{Change in microstructure}

Figure 11 shows a schematic illustration of the change in microstructure after annealing and draw bending. 


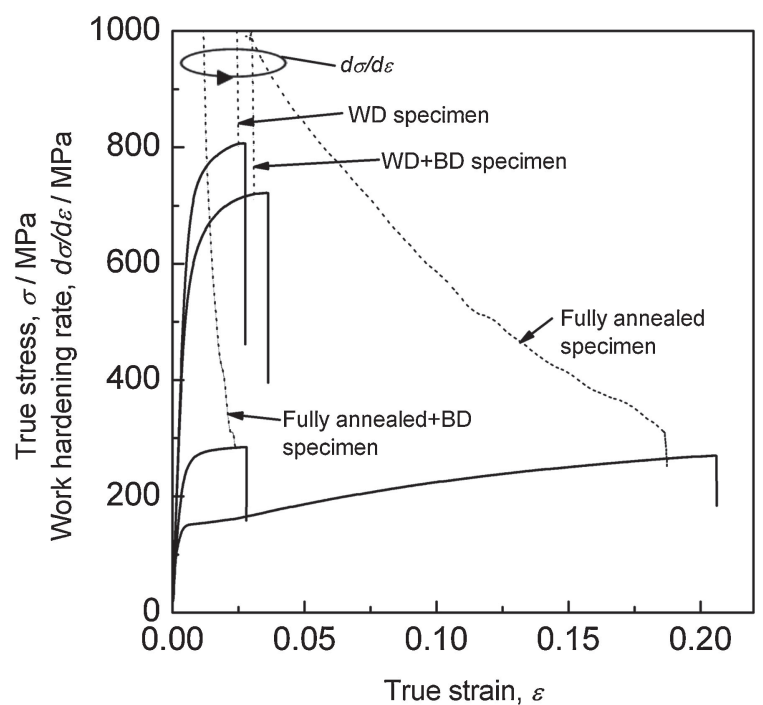

Fig. 10 True stress-strain curves of the CBD processed wire specimens with work hardening rate $(\mathrm{d} \sigma / \mathrm{d} \varepsilon)$.

Firstly, we discuss the microstructure of the wire specimen annealed after WD $\left(\varepsilon^{\mathrm{WD}}=7.2\right)$. The wire specimen processed by WD shows many dislocation substructures and LAGBs in the ultrafine grains. ${ }^{11)}$ In the case of annealing at various temperatures after $\mathrm{WD}$, it was confirmed that the fraction of HAGBs and the grain size increased, and the recovery and recrystallization occurred inside the grains (as shown in Figs. 7 and 8).

Secondly, we discuss the microstructure of the wire specimen after draw bending. During draw bending, the annealed specimens were subjected to compressive/tensile or tensile/compressive strains in the longitudinal direction, as shown in Fig. 5. In the WD and 3/4TS specimens, the dislocations which are piled up by WD could be annihilated by draw bending. It can be considered while draw bending as that collision of dislocations which has opposite sign cause annihilation. On the other hand, in the fully annealed, 1/2EL and $1 / 4 \mathrm{EL}$ specimens, the dislocations may be increased rapidly by draw bending, because there are already no dislocations or fewer dislocation substructures inside the grains. In the $1 / 2 \mathrm{TS}$ specimen, it is considered that the grain size and addendum modification inside grain remains the same before and after draw bending, consequently this mechanical property remains unchanged.

\section{Conclusions}

The mechanical behaviors of $\mathrm{Cu}-\mathrm{Sn}$ alloy wire specimens before and after draw bending, and the evolution of microstructures are systematically investigated. During draw bending, the WD and final annealing specimens are subjected to compressive/tensile or tensile/compressive strains in the longitudinal direction. It is confirmed that the mechanical behaviors are categorize into softening induced by plastic deformation and work hardening. This phenomenon is related to the recovery condition inside the grains produced by heat treatment. Specifically, after draw bending the WD and 3/4TS specimens, the change in grain size is observed to be smaller than the deterioration of strength, and it is confirmed that the fraction of HAGBs increases after the final annealing of the specimens. As the reason for the deterioration of the strengths of the WD and 3/4TS specimens, the draw bending could be introduced dislocations which are piled up by WD to the opposite direction of the WD.

\section{Acknowledgements}

This study was financially supported by a Grant-inAid for Scientific Research on Innovative Area, "Bulk Nanostructured Metals", through MEXT, Japan (contract No. 22102005), which is gratefully appreciated. Yazaki Parts Company is also acknowledged for supplying the copper rods used in this study.

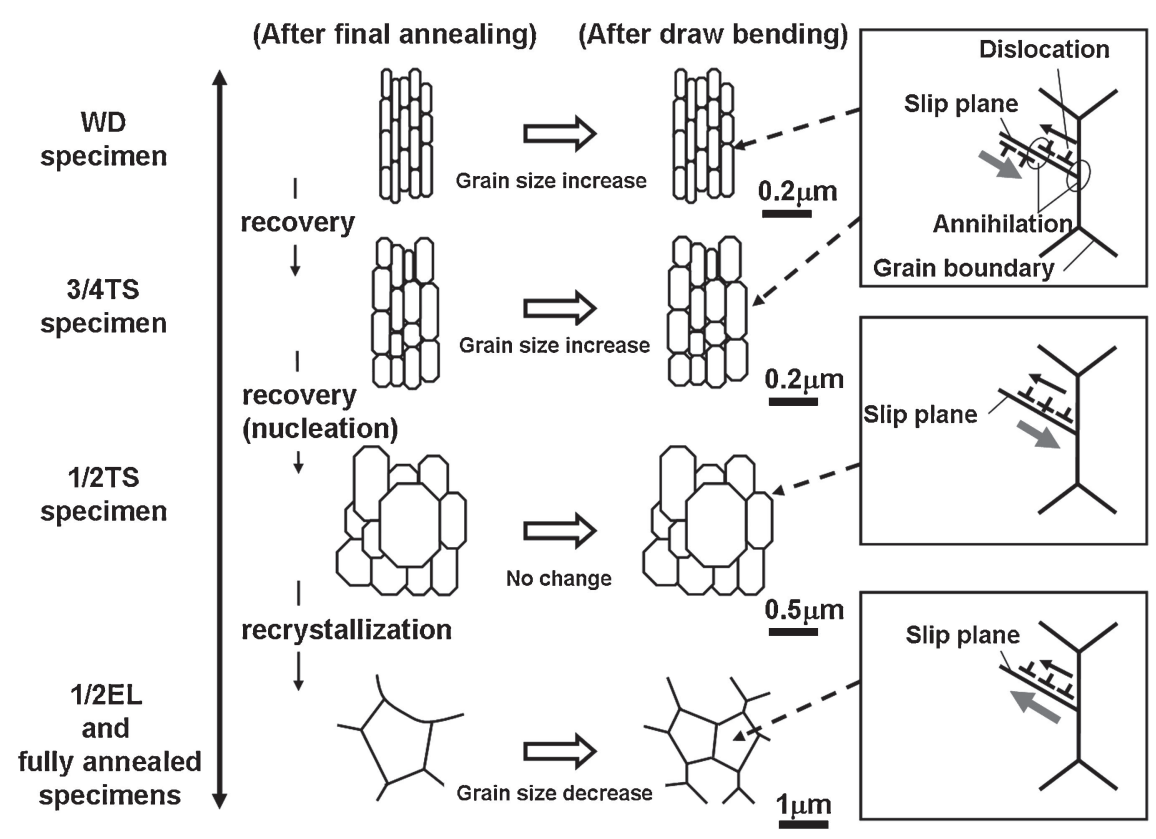

Fig. 11 Schematic illustration showing microstructural evolution after final annealing and draw bending in the copper alloy. 


\section{REFERENCES}

1) R. Z. Valiev, A. V. Korznikov and R. R. Mulyukov: Mater. Sci. Eng. A 168 (1993) 141-148.

2) Y. Ma, M. Furukawa, Z. Horita, M. Nemoto, R. Z. Valiev and T. G. Langdon: Mater. Trans. JIM 37 (1995) 336-339.

3) V. M. Segal: Mater. Sci. Eng. A 197 (1995) 157-164.

4) Z. Horita, M. Furukawa, T. G. Langdon and M. Nemoto: Materia Japan 37 (1998) 767-774.

5) Y. Saito, H. Utsunomiya, N. Tsuji and T. Sakai: Acta Mater. 47 (1999) 579-583.

6) N. Tsuji, Y. Saito, H. Utsunomiya and T. Sakai: Ultra-Fine Grained Materials, ed. by R. S. Mishra, S. L. Semiatin, C. Suryanarayana, N. N. Thadhani and T. C. Low (TMS Warrendale PA, 2000) pp. 207218.

7) N. Tsuji, Y. Saito, H. Utsunomiya and S. Tanigawa: Scr. Mater. 40 (1999) 795-800.
8) N. Tsuji, R. Ueji and Y. Saito: Materia Japan 39 (2000) 961.

9) N. Tsuji, R. Ueji and Y. Minamino: Scr. Mater. 47 (2002) 69-76.

10) A. Azushima, R. Kopp, A. Korhonen, D. Y. Yang, F. Micari, G. D. Lahoti, P. Groche, J. Yanagimoto, N. Tsuji, A. Rosochowski and A. Yanagida: Annal. CIRP 57 (2008) 716-735.

11) K. Hanazaki, N. Shigeiri and N. Tsuji: Mater. Sci. Eng. A 527 (2010) 5699-5707.

12) J. Yanagimoto, J. Tokutomi, K. Hanazaki and N. Tsuji: Annal. CIRP 60 (2011) 279-282.

13) E. O. Hall: Proc. Phys. Soc. B 64 (1951) 747.

14) N. J. Petch: J. Iron steel Inst. 174 (1953) 25.

15) R. W. Armstrong: Trans. Indian Inst. Met. 50 (1997) 521-531.

16) N. Kamikawa, X. Huang, N. Tsuji and N. Hansen: Acta Mater. 57 (2009) 4198-4208

17) Y. Wang, M. Chen, F. Zhou and E. Ma: Nature 419 (2002) 912-914.

18) H. W. Kim, S. B. Kang, N. Tsuji and Y. Minamino: Acta Mater. 53 (2005) 1737-1749. 